\title{
1-Nitronaphtalene as a Dienophile in Diels-Alder Reactions
}

\author{
E. Paredes, B. Biolatto, M. Kneeteman and P. M. Mancini \\ Laboratorio Fester, Dpto. de Química, Facultad de Ingeniería Química, Universidad Nacional del Li- \\ toral, Santiago del Estero 2829, 3000, Santa Fe, Argentina \\ E-mail: pmancini@fiqus.unl.edu.ar
}

\begin{abstract}
: the utilization of substitued dienes with electron-donor groups and under high pressure conditions, induces the dienophilic character of 1-nitronaphtalene in Diels-Alder reactions, giving the products with and without the nitro-group, the yield depending on the nature of the dienes substituent groups.
\end{abstract}

\section{Introduction}

The Diels-Alder reaction has been subject of extensive studies for both theoretical and mechanistic purposes, and because of the synthetic advantages it offers. However, the exploration of this reaction using aromatic compounds as dienophiles is scarce [1], particularly for compounds showing a special stability like in the case of 1-nitronaphthalene [2]. Though the nitro-substituent promotes the dienophilicity of such a compounds, the lack reactivity in reactions with isoprene and other simple dienes has been evident, either under thermal or hyperbar conditions [3]. Therefore, it was interesting the study of reactivity of this dienophile with more complex and reactive dienes like 1-( $N$-acetyl- $N$ propylamino)-1,3-butadiene and 1-methoxy-3-trimethylsilyloxi-1,3-butadiene (Danishefsky diene). They proved to successfully revert the precedent tendency, specially under high pressure conditions, leading to highly functionalyzed adducts.

\section{Experimental}

The Diels-Alder reactions between 1-nitronaphthalene and 1-( $N$-acetyl- $N$-propylamino)-1,3butadiene or 1-methoxy-3-trimethylsilyloxi-1,3-butadiene, were carried out at $40^{\circ} \mathrm{C}$ and $11.5 \mathrm{kbar}$ of pressure, during 53 hours.

\section{Results and Discussion}

The dienophilic character that 1-nitronaphthalene exhibit in the reaction with the Danishefsky diene leads to a mixture of adducts keeping the nitro-substitution, which under the purification conditions suffer aromatization with extrusion of nitrous acid and methanol. (Scheme 1), the dienophile recover 
being 30\% and yield 90\% (based on consumed starting nitronaphtalene). Instead, traces of products are obtained when 1-( $N$-acetyl- $N$-propylamino)-1,3-butadiene is used as diene, concluding that this diene doesn't hold a electrondonor substituent strong enough to induce the 1-nitronaphthalene to react in the expected way. It is worth mentioning in first place, the importance of the study of 1-nitronaphtalene's behavior as dienophile, not exaustively explored, and in second instance, the possibility of synthesis of adducts with the base skeleton of diterpenes through one-step reactions as it is the Diels-Alder<smiles>C=C(C)OC(=C)/C=C/COC(=O)OC</smiles>

reaction.

Acknowledgements: We are indebted to the Science and Technology Secretariat - Universidad Nacional del Litoral, República Argentina, for the financial support, CAI+D Program (96-00-024-161). The authors want to thank Prof. Serge Piettre from IRCOF - Université de Rouen, France, for his valuable advice, and Dr. Gonzalez Sierra from the IQUIOS - UNR (Argentina) for the NMR experiments.

\section{References and Notes}

1. Hurd, C.D.; Juel, L.H. J. Am. Chem. Soc. 1955, 77, 601- 606.

2. Wenkert, E.; Moeller, P. D. R.; Piettre, S. J. Am. Chem. Soc. 1988, 110, 7188-7194.

3. Paredes, E.; Biolatto, B.; Kneeteman, M.; Gonzalez Sierra, M.; Mancini, P. Unpublished results. 